\title{
GRB 020813: Polarization in the case of a smooth optical decay ${ }^{\star}$
}

\author{
J. Gorosabel ${ }^{1,2}$, E. Rol ${ }^{3}$, S. Covino ${ }^{4}$, A. J. Castro-Tirado ${ }^{1}$, J. M. Castro Cerón ${ }^{2}$, D. Lazzati ${ }^{5}$, J. Hjorth ${ }^{6}$, D. Malesani ${ }^{7}$, \\ M. Della Valle ${ }^{8}$, S. di Serego Alighieri ${ }^{8}$, F. Fiore ${ }^{9}$, A. S. Fruchter ${ }^{2}$, J. P. U. Fynbo ${ }^{10}$, G. Ghisellini ${ }^{4}$, P. Goldoni1 ${ }^{11}$, \\ J. Greiner ${ }^{12}$, G. L. Israel ${ }^{9}$, L. Kaper ${ }^{3}$, N. Kawai ${ }^{13}$, S. Klose ${ }^{14}$, C. Kouveliotou ${ }^{15}$, E. Le Floc'h ${ }^{11}$, N. Masetti ${ }^{16}$, \\ F. Mirabel ${ }^{11}$, P. Møller ${ }^{17}$, S. Ortolani ${ }^{18}$, E. Palazzi16, E. Pian ${ }^{19}$, J. Rhoads ${ }^{2}$, G. Ricker ${ }^{20}$, P. Saracco ${ }^{4}$, L. Stella ${ }^{9}$,
} G. Tagliaferri ${ }^{4}$, N. Tanvir' ${ }^{21}$, E. van den $\mathrm{Heuvel}^{3}$, M. Vietri ${ }^{2}$, P. M. Vreeswijk ${ }^{23}$, R. A. M. J. Wijers ${ }^{3}$, and F. M. Zerbi ${ }^{4}$

${ }^{1}$ Instituto de Astrofísica de Andalucía (IAA-CSIC), PO Box 03004, 18080 Granada, Spain

2 Space Telescope Science Institute, 3700 San Martin Drive, Baltimore, MD 21218-2463, USA

3 University of Amsterdam, Kruislaan 403, 1098 SJ Amsterdam, The Netherlands

4 INAF, Osservatorio Astronomico di Brera, via E. Bianchi 46, 23807 Merate (LC), Italy

5 Institute of Astronomy, University of Cambridge, Madingley Road, CB3 OHA Cambridge, UK

6 Astronomical Observatory, University of Copenhagen, Juliane Maries Vej 30, 2100 Copenhagen Ø, Denmark

7 International School for Advanced Studies (SISSA/ISAS), via Beirut 2-4, 34016 Trieste, Italy

8 INAF, Osservatorio Astrofisico di Arcetri, Large E. Fermi 5, 50125 Firenze, Italy

9 INAF, Osservatorio Astronomico di Roma, via Frascati 33, 00044 Monterporzio, Italy

10 Department of Physics and Astronomy, University of Århus, Ny Munkegade, 8000 Århus C, Denmark

11 CEA/DSM/DAPNIA, L'Orme des Merisiers, Bat. 709, 91191 Gif-sur-Yvette, France

12 Max-Planck-Institut für extraterrestrische Physik, 85741 Garching, Germany

13 Department of Physics, Faculty of Science, Tokyo Institute of Technology 2-12-1 Oookayama, Meguro-ku, Tokyo 152-8551, Japan

14 Thüringer Landessternwarte Tautenburg, 07778 Tautenburg, Germany

15 NASA MSFC, SD-50, Huntsville, AL 35812, USA

16 Istituto di Astrofisica Spaziale e Fisica Cosmica, CNR, via Gobetti 101, 40129 Bologna, Italy

17 European Southern Observatory, Karl-Schwarzschild-Straße 2, 85748 Garching, Germany

18 Università di Padova, Dept. di Astronomia, Vicolo dell'Osservatorio 2, 35122 Padova, Italy

19 INAF, Osservatorio Astronomico di Trieste, via G.B. Tiepolo 11, 34131 Trieste, Italy

${ }^{20}$ Center for Space Research, Massachusetts Institute of Technology, Cambridge, MA 02139-4307, USA

21 Department of Physical Sciences, University of Hertfordshire, College Lane, Hatfield, Herts AL10 9AB, UK

${ }^{22}$ Scuola Normale Superiore, Piazza dei Cavalieri, 56100 Pisa, Italy

23 European Southern Observatory, Casilla 19001, Santiago 19, Chile

Received 28 September 2003 / Accepted 23 March 2004

\begin{abstract}
We present the results of a VLT polarimetric monitoring campaign of the GRB 020813 optical afterglow carried out in three nights, from 0.88 to 4.20 days after the gamma-ray event. The mean values of the degree of linear polarization $(P)$ and its position angle $(\theta)$ resulting from our campaign are $\langle P\rangle=1.18 \pm 0.10 \%$ and $\langle\theta\rangle=148.7^{\circ} \pm 2.3^{\circ}$, after correcting for Galactic interstellar polarization. Our VLT data set is most naturally described by a constant degree of linear polarization and position angle, nonetheless a slow $\theta$ evolution cannot be entirely ruled out by our data. The VLT monitoring campaign did not reveal either a significant $\theta$ rotation respect to the Keck spectropolarimetric observations performed $\sim 0.25$ days after the GRB (Barth et al. 2003). However, $\langle P\rangle$ is a factor of two lower than the polarization degree measured from Keck. Additionally, the VLT polarization data allowed us to construct an accurate $V$-band light curve. The $V$-band photometric data revealed a smooth light curve with a break located between the last Keck and our first VLT polarimetric measurement, $0.33<t_{\text {break, } V}<0.88$ days after the GRB. The typical magnitude fluctuations of the VLT $V$-band lightcurve are $0.003 \mathrm{mag}, 0.010 \mathrm{mag}$ and $0.016 \mathrm{mag}$ for our three observing nights, respectively. We speculate that the stability of $\theta$ might be related to the smoothness of the light curve.
\end{abstract}

Key words. gamma rays: bursts - techniques: photometric - techniques: polarimetric

Send offprint requests to: J. Gorosabel, e-mail: jgu@iaa.es

* Based on observations collected at the European Southern Observatory, Cerro Paranal (Chile), ESO programmes 69.D-0461(A) and 69.D-0701(A).

\section{Introduction}

GRB 020813 was detected by the HETE-2 spacecraft on Aug. 13.11411 UT (Villaseñor et al. 2002) as a bright long 
(duration > $125 \mathrm{~s}$; Hurley et al. 2002) gamma-ray burst (GRB) and its position was rapidly disseminated among the GRB community. After the optical afterglow (OA) discovery (Fox et al. 2002) it was subject of an intensive radio (Frail \& Berger 2002; Bremer \& Castro-Tirado 2002), X-ray (Butler et al. 2003), optical (Li et al. 2003; Laursen \& Stanek 2003; Urata et al. 2003), near-IR (Covino et al. 2003a), polarimetric (Barth et al. 2002; Covino et al. 2002) and spectroscopic (Price et al. 2002; Barth et al. 2003) follow-up.

Among the thousands of GRBs detected to date, only $~ 50$ of those accurately localized ${ }^{1}$ have been pinpointed at optical wavelengths. Positive linear polarization detections have been reported for 7 of them, typically at a level of $1-3 \%$ (with the possible exception of the $\sim 10 \%$ polarization reported for GRB 020405 by Bersier et al. 2003). A review on the polarization detections to date can be found in Covino et al. (2003b) and Björnsson (2002). Optical afterglow emission is widely accepted to be synchrotron radiation, the result of the interaction of the GRB relativistic wave with the circumburst medium (fireball model; see Rees \& Mészáros 1992; Mészáros \& Rees 1997). In general, if the fireball configuration is not symmetric, some degree of polarization is expected from the synchrotron radiation.

Several asymmetric scenarios able to account for the $1-3 \%$ polarization typically measured in OAs have been proposed. Among them are: $i$ ) causally disconnected magnetic patches (Gruzinov \& Waxman 1999), ii) a homogeneous collimated fireball observed off-axis (Ghisellini \& Lazzati 1999; Sari 1999) and iii) a similar scenario where the jet is structured (the collimated energy per unit angle decays smoothly with the angle from the jet axis; Rossi et al. 2002).

In the context of $i$ ) $\theta$ and $P$ are expected to change on the same time scale. The latter two scenarios (ii) and iii)) show characteristic evolutions of $\theta$ and $P$ when the outflow slows down and the Lorentz factor decreases. It is especially interesting that scenario $\mathrm{i}$ ) predicts a double-peaked evolution of the degree of polarization, with a polarization angle change of 90 degrees in between the two peaks, while in contrast scheme iii) predicts a single peak of polarized emission, with a constant polarization angle. If an ordered magnetic field exists in the medium into which the shock propagates, this can result in a $\theta$ roughly constant in time, accompanied by a variable $P$ (Granot \& Königl 2003).

Among the 7 positive OA polarization detections there are only two clear cases (GRB 021004 and GRB 030329) where a rotation in the polarization angle has been reported (Rol et al. 2003; Greiner et al. 2003). In both cases the OA shows a complex light curve. So a satisfactory description of the polarization evolution is still pending. In the present paper we report an intensive polarimetric follow up of the GRB 020813 OA. In a companion paper Lazzati et al. (2004) report a physical interpretation of the polarization data published in this study.

\footnotetext{
${ }^{1}$ See http://www .mpe.mpg.de/ jcg/grbgen.html
}

\section{Observations}

The polarimetric follow up observations started 0.8795 days after the gamma-ray event. The observations were performed in the $V$-band with the FOcal Reducer/low dispersion Spectrograph 1 (FORS1) at the Very Large Telescope (VLT), unit 3 . The $2048 \times 2048$ FORS 1 CCD yields a pixel scale of $0.2^{\prime \prime} / \mathrm{pix}$, and was used in the high gain 4-ports readout mode. A Wollaston prism in tandem with a rotatable half-wave retarder allowed us to determine the Stokes $Q$ and $U$ parameters. For each retarder angle $\phi / 2$, two orthogonal simultaneous images with polarization angles $\phi$ and $\phi+90^{\circ}$ were obtained. Our polarimetric data are based on four $\phi / 2$ values $(0.0,22.5,45.0$, and 67.5 degrees), the observations being consecutive executions of four exposure cycles. The log of observations presented in Table 1 contains 33 polarimetric cycles executed during three nights, amounting to $\sim 13 \mathrm{~h}$ of VLT exposure time.

\section{Analysis}

The images were reduced following standard procedures. First, for each of the FORS1 images the chip overscan of the four readout ports was subtracted. This was done because the four pedestal levels vary slightly from one image to another. This procedure was carried out for all the science and calibration images (including the bias images). Then a master bias frame was constructed by median combination and subtracted from the science and flat field images. Finally the science images were divided by a master normalized flat field image. The master flat field image was created combining sky flat field frames that were acquired without the Wollaston prism and the retarder plate in the light path ${ }^{2}$. This procedure was applied separately to the data taken each observing night.

The photometry is based on circular aperture photometry, fixing the radius to the OA Full Width at Half Maximum (FWHM). The results presented in this study remain qualitatively unaltered for aperture radii ranging from 0.5 to 3 times the OA FWHM. Verification of the photometry, calibration, and the reduction procedure, were performed observing each night a polarization standard star; BD-12 5133 (13-14/08/2002), Hiltner 652 (14-15 and 16-17/08/2002). The photometry and the reduction of the standard stars was identical to the one applied to the science images.

The determination of the parameters describing the linear polarization of $(P$ and $\theta)$ is based on the construction of the $S(\phi)$ function for the four $\phi / 2$ retarder angles following the procedure described in Covino et al. (1999). Then a fit of the form $S(\phi)=\frac{P(\%)}{100} \cos 2(\theta-\phi)$ was used to evaluate simultaneously the values of $P(\%)$ and $\theta$. An independent verification of the derived $P$ and $\theta$ values was performed evaluating the Stokes parameters $(Q, U)$ for all the objects in the GRB field based on Fourier transformation arithmetics (see FORS1 manual expressions ${ }^{3}$ ). Both methods yielded consistent results (see Fig. 1).

\footnotetext{
${ }^{2}$ Following the recommendations given in http://www. eso.org/instruments/fors1/pola.html

${ }_{3}$ FORS1+2 manual, ref. VLT-MAN-ESO-13100-1543, posted at http://www. eso.org/instruments/fors/userman/
} 
Table 1. Log of the observations carried out with the VLT(+FORS1). The table is divided in three sub tables, one for each observing night. At the beginning of each night the magnitude corresponding to a short acquisition image $\left(T_{\exp }=20-150 \mathrm{~s}\right)$ is displayed. The polarization images are summarized in blocks of 4 images cycles $\left(4 \times T_{\text {exp }}\right)$ with the FORS1 half wave plate rotator at $0.0,22.5,45.0$ and 67.5 degrees.

\begin{tabular}{|c|c|c|c|c|c|c|}
\hline $\begin{array}{l}\text { Date UT } \\
\text { Aug. } 2002\end{array}$ & $\begin{array}{l}T_{\text {exp }} \\
(\mathrm{s})\end{array}$ & Filter & $\begin{array}{l}\text { Seeing } \\
\left({ }^{\prime \prime}\right)\end{array}$ & Magnitude & $\begin{array}{c}P \\
(\%)\end{array}$ & $\begin{array}{c}\theta \\
\text { (degrees) }\end{array}$ \\
\hline $13.99037-13.99071$ & 30 & $V$ & 0.53 & $20.6560 \pm 0.0190$ & $\#$ & $\#$ \\
\hline $13.99358-14.03042$ & $4 \times 750$ & $V$ & 0.64 & $20.6855 \pm 0.0022$ & $1.07 \pm 0.22$ & $154.3 \pm 5.9$ \\
\hline $14.03233-14.06918$ & $4 \times 750$ & $V$ & 0.72 & $20.7485 \pm 0.0022$ & $1.42 \pm 0.25$ & $137.0 \pm 4.4$ \\
\hline $14.07107-14.10791$ & $4 \times 750$ & $V$ & 0.68 & $20.8070 \pm 0.0022$ & $1.11 \pm 0.22$ & $150.5 \pm 5.5$ \\
\hline $14.11190-14.14869$ & $4 \times 750$ & $V$ & 0.70 & $20.8600 \pm 0.0022$ & $1.05 \pm 0.23$ & $146.4 \pm 6.2$ \\
\hline $14.22276-14.23876$ & $4 \times 300$ & V & 0.84 & $20.9990 \pm 0.0036$ & $1.43 \pm 0.44$ & $155.8 \pm 8.5$ \\
\hline $14.24506-14.26128$ & $4 \times 300$ & $V$ & 0.84 & $21.0355 \pm 0.0036$ & $1.07 \pm 0.53$ & $163.0 \pm 14.6$ \\
\hline $14.26340-14.27943$ & $4 \times 300$ & $V$ & 0.74 & $21.0555 \pm 0.0036$ & $1.37 \pm 0.49$ & $142.1 \pm 8.9$ \\
\hline $15.00746-15.02343$ & $4 \times 300$ & $\bar{V}$ & 0.65 & $21.8081 \pm 0.0085$ & $1.26 \pm 0.34^{\dagger}$ & $164.7 \pm 7.4^{\dagger}$ \\
\hline $15.02526-15.04125$ & $4 \times 300$ & $V$ & 0.73 & $21.8150 \pm 0.0078$ & - & - \\
\hline $15.04323-15.05923$ & $4 \times 300$ & $V$ & 0.74 & $21.8175 \pm 0.0081$ & - & - \\
\hline $15.06114-15.07715$ & $4 \times 300$ & $V$ & 0.74 & $21.8462 \pm 0.0074$ & - & - \\
\hline $15.07915-15.09516$ & $4 \times 300$ & $V$ & 0.68 & $21.8436 \pm 0.0060$ & - & - \\
\hline $15.09722-15.11326$ & $4 \times 300$ & $V$ & 0.59 & $21.8696 \pm 0.0064$ & - & - \\
\hline $15.11512-15.13116$ & $4 \times 300$ & $V$ & 0.60 & $21.8650 \pm 0.0053$ & - & - \\
\hline $15.14428-15.14451$ & 20 & $V$ & 0.55 & $21.8630 \pm 0.0322$ & \# & $\#$ \\
\hline $15.14650-15.16247$ & $4 \times 300$ & $V$ & 0.62 & $21.8870 \pm 0.0071$ & - & - \\
\hline $15.16428-15.18026$ & $4 \times 300$ & $V$ & 0.67 & $21.9229 \pm 0.0067$ & - & - \\
\hline $16.98821-16.98995$ & 150 & $R$ & 0.52 & $22.3220 \pm 0.0605$ & \# & \# \\
\hline $17.01049-17.01223$ & 150 & $V$ & 0.60 & $22.8600 \pm 0.0502$ & $\#$ & $\#$ \\
\hline $17.01398-17.03003$ & $4 \times 300$ & $V$ & 0.66 & $22.8986 \pm 0.0271$ & $0.58 \pm 1.08^{\star}$ & $13.7 \pm 24.4^{\star}$ \\
\hline $17.03193-17.04800$ & $4 \times 300$ & $V$ & 0.69 & $22.9036 \pm 0.0237$ & - & - \\
\hline $17.05027-17.06634$ & $4 \times 300$ & $V$ & 0.84 & $22.9500 \pm 0.0361$ & - & - \\
\hline $17.06817-17.08429$ & $4 \times 300$ & $V$ & 0.87 & $22.8842 \pm 0.0255$ & - & - \\
\hline $17.08614-17.10224$ & $4 \times 300$ & $V$ & 0.82 & $22.8863 \pm 0.0240$ & - & - \\
\hline $17.10488-17.12100$ & $4 \times 300$ & $V$ & 0.83 & $22.9385 \pm 0.0248$ & - & - \\
\hline $17.12294-17.13906$ & $4 \times 300$ & $V$ & 0.84 & $22.9307 \pm 0.0260$ & - & - \\
\hline $17.14094-17.15707$ & $4 \times 300$ & $V$ & 0.73 & $22.9031 \pm 0.0245$ & - & - \\
\hline $17.15920-17.17533$ & $4 \times 300$ & $V$ & 0.88 & $22.9339 \pm 0.0290$ & - & - \\
\hline $17.17720-17.19334$ & $4 \times 300$ & $V$ & 0.80 & $22.9127 \pm 0.0234$ & - & - \\
\hline $17.19529-17.21146$ & $4 \times 300$ & $V$ & 0.78 & $22.9402 \pm 0.0243$ & - & - \\
\hline $17.21335-17.22953$ & $4 \times 300$ & $V$ & 0.81 & $22.9716 \pm 0.0266$ & - & - \\
\hline $17.23241-17.24859$ & $4 \times 300$ & $V$ & 0.70 & $22.9640 \pm 0.0261$ & - & - \\
\hline $17.25048-17.26666$ & $4 \times 300$ & $V$ & 0.78 & $22.9646 \pm 0.0234$ & - & - \\
\hline $17.26900-17.28521$ & $4 \times 300$ & $V$ & 0.88 & $22.9595 \pm 0.0194$ & - & - \\
\hline $17.28713-17.30334$ & $4 \times 300$ & $V$ & 1.02 & $22.9752 \pm 0.0212$ & - & - \\
\hline $17.30525-17.32148$ & $4 \times 300$ & V & 1.00 & $23.0218 \pm 0.0244$ & - & - \\
\hline
\end{tabular}

\# Not applicable.

$\dagger$ Value obtained when co-adding all the polarimetric images taken on Aug. 15.00746-15.18026 UT.

$\star$ Value obtained when co-adding all the polarimetric images taken on Aug. 17.01398-17.32148 UT.

The Galactic interstellar Medium (ISM) reddening in the direction of GRB 020813 is not negligible $(E(B-V)=$ $0.111 \mathrm{mag}$; Schlegel et al. 1998), so it induces (approximately) a systematic offset for all the field objects in the Stokes $(Q, U)$ plane. Thus, in order to compensate the effect of the ISM, the weighted mass center of the $(Q, U)$ distribution was calculated for 20 stars in the field and shifted to the Stokes plane origin. We realized that stars located close to the border of the FORS1 chip show a residual polarization once the interstellar polarization effect has been corrected (see upper panel of Fig. 1). Thus, stars located further than $1.7^{\prime}$ from the image center were not used for the Galactic ISM polarization correction. The introduced mean offset is given by $\Delta Q_{\text {ISM }}=-6.22 \pm 0.59 \times 10^{-3}$ and $\Delta U_{\text {ISM }}=3.95 \pm 0.80 \times 10^{-4}$. These values correspond to $\theta_{\text {ISM }}=178.2^{\circ} \pm 2.6^{\circ}$ and $P_{\text {ISM }}=$ $0.62 \pm 0.06 \%$, in agreement with the ISM correction carried out by Covino et al. (2002): $\theta_{\mathrm{ISM}}=178^{\circ}$ and $P_{\mathrm{ISM}}=0.59 \%$. We have verified that for the applied $\left(\Delta Q_{\mathrm{ISM}}, \Delta U_{\text {ISM }}\right)$ mean 

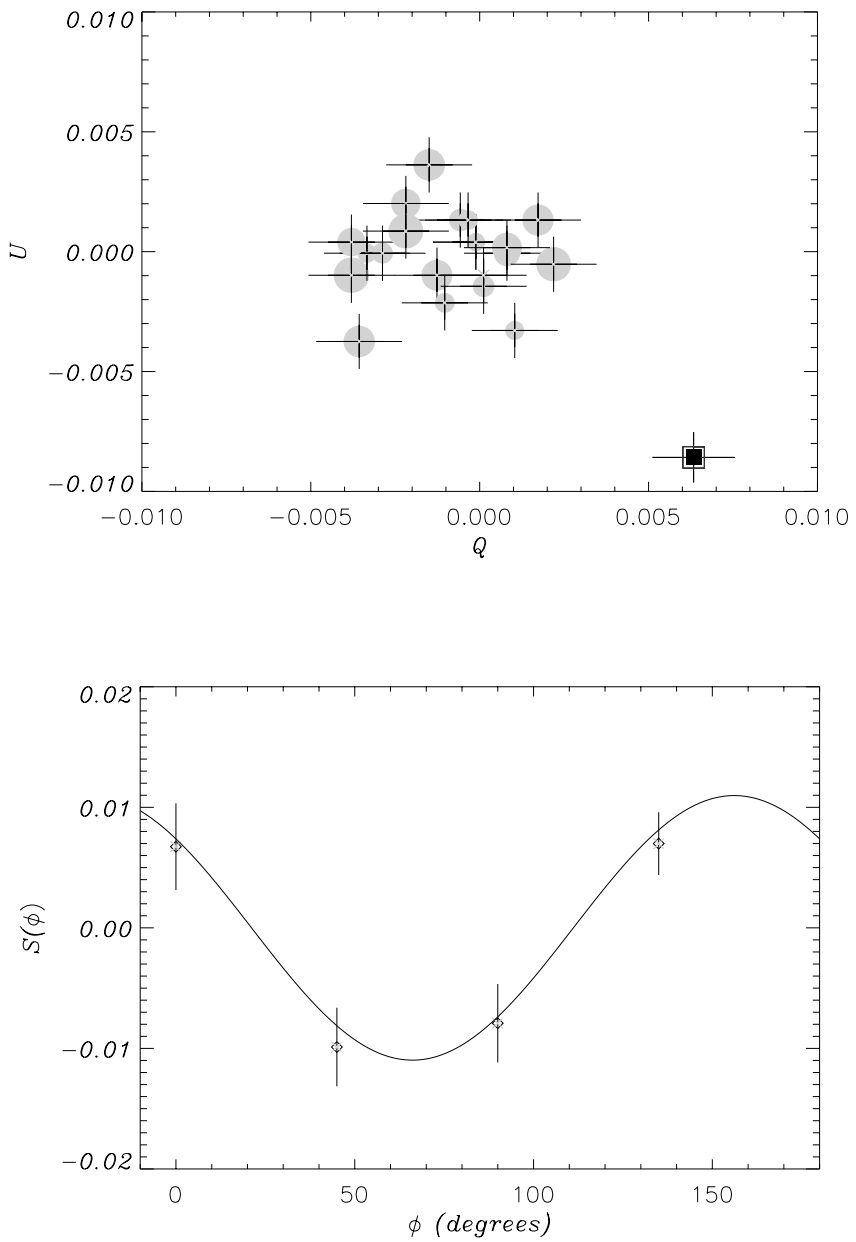

Fig. 1. Both plots correspond to the observations done on Aug. 13.99358-14.03042 UT $\left(T_{\exp }=4 \times 750 \mathrm{~s}\right)$. Upper panel: the diagram shows the Stokes $(Q, U)$ parameters of 20 field stars (circles) and the OA (square), once the ISM correction has been introduced. The OA is clearly polarized, located away from the diagram stellar population. The circle sizes are proportional to the distance of each star from the center of the FORS1 image. It was noticed that the distance from the stars to the OA (placed in the center of the FORS1 CCD) is correlated to their polarization, presumably due to a residual instrumental polarization effect. Thus, stars further than 1.7' (not included in the plot) from the CCD center were not considered for the ISM correction. Lower panel: the plot displays the $S(\phi)=\frac{P(\%)}{100} \cos 2(\theta-\phi)$ fit in agreement with the OA position in the upper plot. The fit yields $P=1.07 \pm 0.22 \%, \theta=154.3^{\circ} \pm 5.9^{\circ}$, with $\chi^{2} /$ d.o.f. $=0.07$.

offset the field stars remain, at different epochs, at the same position (within errors) on the Stokes plane. Empirical relations (Serkowski, Mathewson \& Ford 1975) indicate that $P_{\mathrm{ISM}}(\%) \leq$ $9 \times E(B-V) \mathrm{mag}$, in agreement with the derived $P_{\text {ISM }}$ in the direction of GRB 020813.

Once the ISM correction is introduced, the value of $P$ has been multiplied by $\sqrt{1-\left(\sigma_{P} / P\right)^{2}}, \sigma_{P}$ being the standard deviation of $P$. This correction factor is introduced because $P$ is positive by definition, so averaged low signal-to-noise $(\mathrm{S} / \mathrm{N})$ polarization estimates (as in our case) tend to overestimate the actual value of $P$ (di Serego Alighieri 1997). Although FORS1 uses a super-achromatic half-wave plate, it introduces an offset in the $V$-band polarization angle of $1.80^{\circ}$, which has to be subtracted from the inferred $\theta$ values.

\section{Results and discussion}

\subsection{Determination of the break time}

The accurate determination of the light curve break has an important impact on the interpretation of the polarimetric evolution. However, the published positions of the $V$-band break time $\left(t_{\text {break }, V}\right)$ are not in close agreement. Thus, Urata et al. (2003) report $t_{\text {break, } V}=0.22 \pm 0.01$ days, whereas Covino et al. (2003a) give $t_{\mathrm{break}, V}=0.50 \pm 0.27$ days. In addition, $\mathrm{Li}$ et al. (2003) determined an $R$-band break time at $t_{\text {break, } R}=$ $0.14 \pm 0.03$ based on data of the KAIT telescope. Again this break time is not consistent with the $R$-band break time ( $t_{\text {break }, R}=0.57 \pm 0.05$ days) derived by Covino et al. (2003a). Therefore, it is not clear whether the spectropolarimetric Keck observations carried out 0.19-0.33 days after the GRB (Barth et al. 2003) were acquired after, during or before the break.

Given the relevance of a proper $t_{\text {break, } V}$ determination, we have enhanced our VLT sample with the published GRB 020813 V-band magnitudes to date (Di Paola et al. 2002; Covino et al. 2003a; Urata et al. 2003) and fitted a smoothly broken power law ${ }^{4}$ (Beuermann et al. 1999). At this point no host galaxy contribution was considered in the fit, repeating exactly the procedure previously carried out by other authors for GRB 020813 (Covino et al. 2003a; Urata et al. 2003). The compiled $V$-band data points were shifted to our VLT photometric zero point (see Sect. 4.2). The fit yields $t_{\text {break, } V}=$ $0.56 \pm 0.21$ days, in agreement with the results by Covino et al. (2003a; $t_{\text {break }, V}=0.50 \pm 0.27$ days).

We have checked the impact that the host galaxy contribution ( $\sim 25 \%$ of the total $V$-band flux of our last VLT data points) might have on the fit and therefore on the $t_{\mathrm{break}, V}$ determination. For this purpose two additional late epoch data points (where the host is dominant) were added to our data set. First, the $B R$-band data points acquired with the $1.54 \mathrm{~m}$ Danish Telescope $(1.54 \mathrm{dk}) \sim 58.5$ days after the burst (Gorosabel et al. 2002) were interpolated in the $V$-band yielding $V=24.2 \pm 0.2$. Second we included in our fits the HST/ACS $V$-band magnitude $(V=24.4 \pm 0.2)$ based on the observations carried out 70.03 days after the GRB event ${ }^{5}$. In this case the smoothly broken power law fit yields $t_{\text {break }, V}=0.46 \pm 1.09$ days, indicating the lack of enough $V$-band data for a simultaneous fit in the entire parameter space (given by $k_{1}, k_{2}, \alpha_{1}, \alpha_{2}, s$ and $V_{\text {host }}$ ).

In order to reduce the number of parameters in the fit $s=1$ was fixed, as previously carried out for other OAs (Israel et al. 1999; Stanek et al. 1999). Then, leaving $V_{\text {host }}$ as a free parameter, the fit is improved $\left(\chi^{2} /\right.$ d.o.f. $\left.=1.22\right)$, yielding; $\alpha_{1}=-0.55 \pm 0.09, \alpha_{2}=-1.75 \pm 0.08, t_{\text {break }, V}=0.57 \pm 0.11$ days,

${ }^{4} F_{v}=\left(F_{1}^{-s}+F_{2}^{-s}\right)^{-1 / s}$, with $F_{i}=k_{i} t^{\alpha_{i}}$ being the pre $(i=1)$ and post $(i=2)$ break power law decays, and $s$ a non negative number. In this formalism $t_{\text {break }}=\left(\frac{k_{1}}{k_{2}}\right)^{\frac{1}{\alpha_{2}-\alpha_{1}}}$.

5 A detailed analysis on the light curve and the HST observations (Based on data acquired under Cycle 11 programme \#9405, P.I.: Fruchter, A.S.) is beyond the scope of the present paper, and will be published elsewhere (Castro Cerón et al. 2004). 


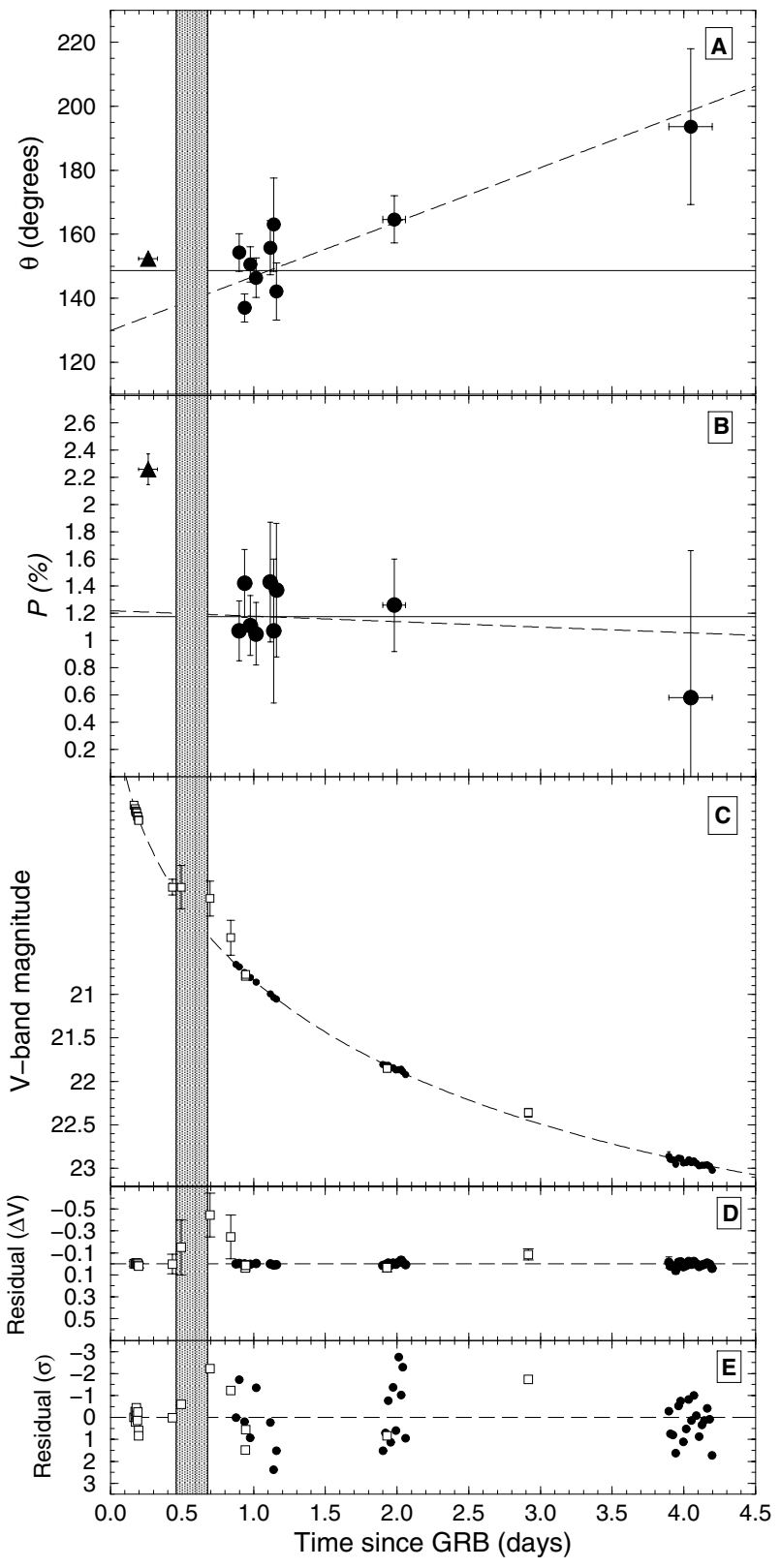

Fig. 2. A) Evolution of the polarization angle $(\theta)$ as a function of time. The circles represent our measurements and the triangle the averaged Keck data point. The width of the error bars indicate the duration of the observations. The solid horizontal line shows the weighted VLT mean and the dashed line the linear fit to the VLT points. B) Linear polarization degree $(P)$, for the same points shown in the upper panel. The horizontal and dashed lines represent the same as in the upper plot. C) V-band light curve, based on the 36 VLT individual data points displayed in Table 1 and on the measurements reported in the literature. The dashed line shows the smoothly broken power law fit when $s=1$. D) $V$-band magnitude residuals when the fitted light curve is subtracted. E) The residuals expressed in units of standard deviations. General: The vertical shaded area shows the break time $1 \sigma$ uncertainty region ( $s=1, t_{\text {break }, V}=0.57 \pm 0.11$ days). The filled circles represent the VLT measurements and the empty squares the data points taken from the literature.

and $V_{\text {host }}=24.61 \pm 0.17$ (see Fig. 2 panel C). The inferred $t_{\text {break }, V}$ value again supports the $V$-band break time reported by Covino et al. (2003a), reducing its error by a factor of $\sim 3$. The shaded vertical band of Fig. 2 indicates the $1 \sigma$ uncertain region of $t_{\text {break, } V}$ when $s=1$. The exercise was repeated for $s>1$ values, which implied higher $t_{\text {break }, V}$ and $\chi^{2} /$ d.o.f. values, hence degrading the fits. In any case for any reasonable value of $s(1<s<30)$ the inferred break time is $t_{\text {break, } V}<0.8795$ days, epoch when our VLT polarimetric observations started. For $s>30$ values the fits progressively disagree with the data $\left(\chi^{2} /\right.$ d.o.f. $\left.>2.0\right)$.

Thus we conclude that, independently of the host galaxy magnitude and the $s$ value, the Keck observations (Barth et al. 2003) were taken before $t_{\text {break, } V}$. Therefore, the $V$-band break time occurred between the last Keck and the first VLT data points, $0.33<t_{\text {break, } V}<0.88$ days after the GRB. This result is in disagreement with the earlier $t_{\text {break, } V}$ determinations inferred by Urata et al. (2003) and also with the $R$-band break epoch derived by Li et al. (2003).

\subsection{The smooth V-band light curve}

In order to minimize the relative magnitude offset present between our $V$-band data points, we have fixed the photometric zero point with respect to a bright unsaturated star detected at high $\mathrm{S} / \mathrm{N}$ ratio level (statistical photometric errors $<0.001)$. The zero point has been tied to the star located at $\alpha_{\mathrm{J} 2000}=19^{\mathrm{h}} 46^{\mathrm{m}} 40.88^{\mathrm{s}}, \delta_{\mathrm{J} 2000}=-19^{\circ} 35^{\prime} 15.9^{\prime \prime}$ which has $V=18.535 \pm 0.008$ (Henden 2002). Thus, our light curve can be easily shifted to other $V$-band zero points. The magnitude errors, displayed in Table 1, include the statistical photometric error of the reference star.

We have analyzed the fluctuations of the $V$-band light curve subtracting from the VLT data points the function fitted in Sect. $4.1(s=1)$. The residuals displayed in Fig. 2 panel D show that the VLT points (filled circles) follow an extremely smooth light curve, even considering the small photometric errors of the first night. The typical magnitude deviations of the VLT data points from the fitted function for our three observing nights are $0.003 \mathrm{mag}, 0.010 \mathrm{mag}$ and $0.016 \mathrm{mag}$, respectively.

For completeness we have overplotted in Fig. 2 (see the open squares of panels $\mathrm{C}, \mathrm{D}$ and $\mathrm{E}$ ) the $V$-band measurements reported by other authors (Di Paola et al. 2002; Covino et al. 2003a; Urata et al. 2003). These points are based on different telescopes/detectors so very likely are not free of relative colour term offsets. Moreover, we can not assure that they were obtained using one consistent photometric technique, so in principle they are expected to be more scattered than our homogeneous VLT data set.

Considering all the photometric measurements (VLT + other authors), the maximum residual corresponds to a $2.74 \sigma$ deviation, associated to the VLT image taken on 15.11512-15.13116 UT (see Fig. 2 panel E). These results suggest that the smooth light curve measured 3.9-4.9 h after the burst (see Laursen \& Stanek 2003) continued at least until 4.2 days after the GRB event. 


\subsection{Polarization monitoring}

Barth et al. (2003) reported a potential rapid evolution of $P$ between $0.19-0.33$ days after the GRB in the 4500-5500 $\AA$ spectropolarimetric wavelength interval. However, this evolution vanishes in the 5800-6800 $\AA$ bin, so these authors caution against any overinterpretation on a possible wavelength dependent $P$ variation (Barth 2003). The FORS1 $V$-band filter transmission curve is centered at $5540.0 \pm 557.5 \AA$, just in the 5500-5800 $\AA$ gap present between the two spectropolarimetric bins. Thus, in order to extract a $V$-band filtered polarimetric data point from the spectropolarimetric data, we have averaged the $P, \theta$ values by Barth et al. (2003) reported for the two wavelength ranges. The averaged $P, \theta$ values have been derived using as a weight the area of the $V$-band filter response curve in the two spectropolarimetric bins ${ }^{6}$. Furthermore given the unclear time evolution of $P$, the three epoch data points by Barth et al. (2003) have been averaged in time. Finally, for consistency we corrected the averaged $(P, \theta)$ values for the slightly different ISM correction applied by Barth et al. (2003) in comparison to our VLT ISM correction ${ }^{7}$. We obtain mean values of $\left\langle P_{\text {Barth }}\right\rangle=2.26 \pm 0.11 \%,\left\langle\theta_{\text {Barth }}\right\rangle=152.4^{\circ} \pm 1.4^{\circ}$ for the Keck observations.

The results of our monitoring campaign are displayed in Table 1. Given the insufficient $\mathrm{OA} S / \mathrm{N}$ ratio in the individual frames taken on 14-15/08/2002 and 16-17/08/2002, the data taken within these two nights have been co-added resulting in two single polarimetric data points. We have reported in panels A and B of Fig. 2 our nine polarimetric measurements (filled circles) along with the mean Keck data point (triangle). The VLT polarimetric points might suggest a slowly increasing trend of $\theta$ with time $(t)$. A linear regression to $\theta(t)$ provides a satisfactory fit $\left(\chi^{2} /\right.$ d.o.f. $\left.=1.34\right)$ consistent with a slope of $17.0 \pm 5.6$ degree/day. Thus, the zero slope constant line (see horizontal solid line of Fig. 2 panel A) departs at a $\sim 3 \sigma$ level from the linear fit (dashed line). However, two arguments point against the suggestive smooth $\theta$ variation.

First, our last inferred value of $\theta$ (Aug. 17.0139817.32148 UT) is based on a measurement which is consistent with no polarization (see Fig. 2 panel B). Second, the fitted linear $\theta$ evolution predicts for the Keck mean observing epoch (triangle of Fig. 2 panel A) $\theta=134.3^{\circ} \pm 6.8^{\circ}$, underestimating the mean Keck polarization angle $\left(\left\langle\theta_{\text {Barth }}\right\rangle=152.4^{\circ} \pm 1.4^{\circ}\right)$ at a $2.6 \sigma$ level. In contrast, a constant polarization angle scenario would naturally match with the three $\theta$ values reported by Barth et al. (2003). The polarization degree measured for the nine VLT points is fully consistent with no evolution, since a linear fit to $P(t)$ yields a slope of $-0.04 \pm 0.25$ (see dashed line of Fig. 2 panel B). Thus, our VLT data are more difficult to accommodate in contexts where $P(t)$ and/or $\theta(t)$ suffer violent rapid fluctuations. However, we cannot exclude that between

\footnotetext{
${ }^{6}$ Assuming a $S(\lambda) \sim \exp ^{-\ln (2)\left(\frac{\lambda-5540}{557.5}\right)^{2}}$ Gaussian profile, the weights were proportional to $\int_{4500 \AA}^{5500 \AA} S(\lambda) \mathrm{d} \lambda$ and $\int_{5800 \AA}^{6800 \AA} S(\lambda) \mathrm{d} \lambda$, respectively.

${ }^{7}$ Barth et al. (2003) applied an ISM correction given by $P_{\mathrm{ISM}}^{\mathrm{Keck}}=$ $0.67 \pm 0.01 \%$ and $\theta_{\mathrm{ISM}}^{\mathrm{Keck}}=167.0^{\circ} \pm 0.3^{\circ}$, in reasonable agreement with $P_{\mathrm{ISM}}^{\mathrm{VLT}}=0.62 \pm 0.06 \%$ and $\theta_{\mathrm{ISM}}^{\mathrm{VLT}}=178.2^{\circ} \pm 2.6^{\circ}$ used in the present work.
}

the epoch of our data points (especially in the gap between Aug. 15.18026 UT and Aug. 17.01398 UT) there might have occurred episodes of rapid variations in $P(t)$ and/or $\theta(t)$.

Thus, within the accuracy, sampling, and coverage of our VLT data, we can claim that there is no rotation of 90 degrees with respect to the polarization angle reported before the light curve break (Barth et al. 2002, 2003) as predicted in the context of several theoretical models (i.e. the nonspreading homogeneous jet, Ghisellini \& Lazzati 1999).

Qualitatively several models might be accommodated in the framework of the Keck plus VLT data set: $i$ ) the lack of polarimetric data $\sim 0.5$ days after the burst does not allow us to exclude a potential $P(t)$ peak coincident with the light curve break. Hence, the single $P(t)$ peak predicted by Rossi et al. (2002) might still be consistent with a $P(t)$ maximum placed between the last Keck and our first VLT data point. $i$ ) If the jet axis was close to the observer line of sight, then also a laterally spreading homogeneous jet might show a single $P(t)$ peak placed at the light curve break, that might agree with the joint data (Sari 1999). iii) A third model potentially compatible with the whole data set might be the one based on a dominant largescale ordered magnetic field, which would exist in the ISM where the shock propagates (Granot \& Königl 2003). In this case a variable $P(t)$ is accompanied by a roughly constant $\theta(t)$, as seen in GRB 020813.

\section{Conclusion}

To date, GRB polarimetric light curves have been sparsely sampled, especially after the break. Several OAs have shown small scale rapid optical fluctuations with respect to the canonical power law decay, but very few have been monitored polarimetrically.

The only two afterglows which have shown a clear $\theta$ evolution to date (GRB 021004, Rol et al. 2003; GRB 030329, Greiner et al. 2003), are accompanied by structured light curves, showing clear deviations from smooth optical decays (Holland et al. 2003; Guziy et al. 2004; Lipkin et al. 2003). In contrast, the GRB 020813 optical afterglow shows a highly smooth light curve and a constant polarization angle, as it has been reported in the present work. It is suggestive to speculate whether the smoothness of the light curve might be correlated with the stability of $\theta(t)$. This speculation is only based on the three mentioned cases, so a further statistical verification would require an intensive polarization monitoring for a significant sample of OAs.

We refer to the companion paper by Lazzati et al. (2004) for a detailed theoretical discussion and interpretation of the data reported in the present work.

Acknowledgements. We thank Aaron Barth for valuable information on the reduction of the GRB 020813 spectropolarimetric data. The authors acknowledge benefits from collaboration within the Research Training Network "Gamma-Ray Bursts: an Enigma and a Tool", funded by the EU under contract HPRN-CT-2002-00294. We acknowledge our anonymous referee for his/her useful comments. 


\section{References}

Barth, A. J., Cohen, M. H., Goodrich, R. W., et al. 2002, GCN Circ. \#1477

Barth, A. J., Sari, R., Cohen, M. H., et al. 2003, ApJ, 584, L47

Barth, A. J. 2003, priv. comm.

Bersier, D., McLeod, B., Garnavich, P. M., et al. 2003, ApJ, 583, L63

Beuermann, K., Hessman, F. V., Reinsch, K., et al. 1999, A\&A, 352, L26

Björnsson, G. 2002, Published in the Proc. of the 1st Niels Bohr Summer Institute on Beaming and Jets in Gamma Ray Bursts, Copenhagen, Denmark, 12-30 Aug. 2002, eConf C0208122:66-72, [arXiv:astro-ph/0302177]

Bremer, M., \& Castro-Tirado, A. J. 2002., GCN Circ. \#1487

Butler, N. R., Marshall, H. L., Ricker, G. R., et al. 2003, ApJ, 597, 1010

Castro Cerón, J. M., et al. 2004, A\&A, in preparation

Covino, S., Lazzati, D., Ghisellini, G., et al. 1999, A\&A, 384, L1

Covino, S., Malesani, D., Ghisellini, G., et al. 2002, GCN Circ. \#1498

Covino, S., Malesani, D., Tavecchio, F., et al. 2003a, A\&A, 404, L5

Covino, S., Ghisellini, G., Lazzati, D., \& Malesani, D. 2003b, in Gamma Ray Burst in the Afterglow Era - Third Workshop, Rome 2002, ASP Conf. Ser., in press [arXiv: astro-ph/0301608]

Di Paola, A., Arkharov, A., Larionov, V., et al. 2002, GCN Circ.\#1505

di Serego Alighieri, S. 1997, in Instrumentation for Large Telescopes, VII Canary Islands Winter School of Astrophysics, ed. J. M. Rodríguez Espinosa, A. Herrero, \& F. Sánchez (Cambridge: Cambridge University Press), 287

Fox, D. W., Blake, C., \& Price, P. A. 2002, GCN Circ. \#1470

Frail, D. A., \& Berger, E. 2002, GCN Circ. \#1490

Ghisellini, G., \& Lazzati, D. 1999, MNRAS, 309, L7

Gorosabel, J., Fynbo, J. P. U., Hjorth, J., et al. 2002, GCN Circ. \#1651

Granot, J., \& Königl, A. 2003, ApJ, 594, L83
Greiner, J., Klose, S., Reinsch, K., et al. 2003, Nature, 426, 157

Gruzinov, A., \& Waxman, E. 1999, ApJ, 511, 852

Guziy, S., Castro-Tirado, A. J., Gorosabel, J., et al. 2004, A\&A, in preparation

Henden, A. A. 2002, GCN Circ. \#1503

Holland, S. T., Weidinger, M., Fynbo, J. P. U., et al. 2003, AJ, 125, 2291

Hurley, K., Cline, T., Mazets, E., et al. 2002, GCN Circ. \#1483

Israel, G. L., Marconi, G., Covino, S., et al. 1999, A\&A, 348, L5

Lazzati, D., et al. 2004, A\&A, 422, 121

Laursen, T. L., \& Stanek, K. Z. 2003, ApJ, 597, L197

Li, W., Filippenko, A. V., Chornock, R., \& Jha, S. 2003, PASP, 115, 844

Lipkin, Y. M., Ofek, E. O., Gal-Yam, A., et al. 2003, ApJ, in press [arXiv:astro-ph/0312594]

Mészáros, P., \& Rees, M. J. 1997, MNRAS, 476, 232

Price, P. A., Bloom, J. S., Goodrich, R. W., et al. 2002, GCN Circ. \#1475

Rees, M. J., \& Mészáros, P. 1992, MNRAS, 258, 41

Rol, E., Wijers, R. A. M. J., Fynbo, J. P. U., et al. 2003, A\&A, 405, L23

Rossi, E., Lazzati, D., Salmonson, J. D., \& Ghisellini, G. 2002, Published in the Proc. of the 1st Niels Bohr Summer Institute on Beaming and Jets in Gamma Ray Bursts, Copenhagen, Denmark, 12-30 Aug., eConf C0208122:88-94, [arXiv: astro-ph/0211020]

Sari, R. 1999, ApJ, 524, L43

Schlegel, D. J., Finkbeiner, D. P., \& Davis, M. 1998, ApJ, 500, 525

Serkowski, K., Mathewson, D. S., \& Ford, V. L. 1975, ApJ, 196, 261

Stanek, K. Z., Garnavich, P. M., Kaluzny, J., Pych, W., \& Thompson, I. 1999, ApJ, 522, L39

Urata, Y., Nishiura, S., Miyata, T., et al. 2003, ApJ, 595, L21

Villaseñor, J., Graziani, C., Shirasaki, Y., et al. 2003, GCN Circ. \#1471 